\title{
Diamond stabilization of ice multilayers at human body temperature
}

\section{Citation}

Wissner-Gross, Alexander D., and Efthimios Kaxiras. 2007. "Diamond Stabilization of Ice Multilayers at Human Body Temperature." Physical Review E 76 (2). https://doi.org/10.1103/ physreve.76.020501.

\section{Permanent link}

http://nrs.harvard.edu/urn-3:HUL.InstRepos:41384103

\section{Terms of Use}

This article was downloaded from Harvard University's DASH repository, and is made available under the terms and conditions applicable to Other Posted Material, as set forth at http:// nrs.harvard.edu/urn-3:HUL.InstRepos:dash.current.terms-of-use\#LAA

\section{Share Your Story}

The Harvard community has made this article openly available.

Please share how this access benefits you. Submit a story.

Accessibility 


\title{
Diamond stabilization of ice multilayers at human body temperature
}

\author{
Alexander D. Wissner-Gross ${ }^{1}$ and Efthimios Kaxiras ${ }^{1,2}$ \\ ${ }^{1}$ Department of Physics, Harvard University, Cambridge, Massachusetts 02138, USA \\ ${ }^{2}$ School of Engineering and Applied Sciences, Harvard University, Cambridge, Massachusetts 02138, USA
}

(Received 13 February 2007; published 27 August 2007)

\begin{abstract}
Diamond is a promising material for wear-resistant medical coatings. Here we report a remarkable increase in the melting point of ice resting on a diamond (111) surface modified with a submonolayer of $\mathrm{Na}^{+}$. Our molecular dynamics simulations show that the interfacial ice bilayer melts at a temperature $130 \mathrm{~K}$ higher than in free ice, and relatively thick ice films $(2.6 \mathrm{~nm}$ at $298 \mathrm{~K}$ and $2.2 \mathrm{~nm}$ at $310 \mathrm{~K})$ are stabilized by dipole interactions with the substrate. This unique physical effect may enable biocompatibility-enhancing ice overcoatings for diamond at human body temperature.
\end{abstract}

DOI: 10.1103/PhysRevE.76.020501

PACS number(s): 64.70.Dv, 61.20.Ja, 61.50.Lt, 61.66.Fn

Recent advances in the synthesis of large-area diamond and diamondlike carbon films are opening up new applications for wear-resistant prosthetics [1-3], artificial heart valves [4], and joint replacements $[5,6]$. While the wear resistance of diamond is dramatically superior to titanium and stainless steel, its blood clotting response as measured by fibrinogen adhesion is slightly worse than these materials [7]. Moreover, the possibility has been raised that nanostructured surface features of diamond might abrade tissue [8]. In the present work, we show that chemically modified diamond (111) films can stabilize remarkably long-ranged orderings of ice up to human body temperatures and at ambient pressure. The soft and hydrophilic ice multilayers sustained by this unique physical effect might enable diamond-coated medical devices that reduce abrasion and are highly resistant to protein absorption [8].

Low-temperature and short-range interfacial ordering of water has been previously predicted and observed on a variety of other planar substrates, including muscovite mica [9-11], platinum [12], chlorine-terminated silicon [13], quartz $[14,15]$, and graphite [16]. Ordering on these substrates has been attributed to electrofreezing [16], lattice matching to partially charged surface atoms [9-11,13,15], polar binding of the first monolayer [12], confinement effects [14], and combined electrofreezing and confinement [17]. Of these mechanisms, only lattice matching to surface ions (in particular, exposed $\mathrm{K}^{+}$ions on muscovite mica) has been demonstrated to passively sustain ordering on an open surface at room temperature [10]. Yet, even the ice phase on mica is not stable when thicker than a single bilayer. Molecular dynamics simulations [18] show a first-order freezing transition for water films of bilayer thickness in nanopores, but for five or six layers there is no transition. Specular x-ray reflection studies of mica (001)-water interfaces [19] reveal a density oscillation amplitude significantly reduced beyond $3 \AA$, while recent Monte Carlo simulations [20] suggest that an ice Ih layer may not even exist at this interface.

In order to stabilize ice coatings with the nanometer-scale thicknesses relevant to macromolecular adsorption, at temperatures relevant to in vivo applications, a novel substrate is therefore needed. In particular, for a stronger ordering effect without external fields and at ambient conditions, a more stable surface chemistry than silicate-intercalated ions is required: in the case of mica, at least half of the surface $\mathrm{K}^{+}$ counterions may be removed by rinsing and replaced with $\mathrm{H}_{3} \mathrm{O}^{+}[19,20]$. The counterions are displaced at two distinct distances from the surface and none remains on the surface, suggesting that the relatively weak solvation efficiency of $\mathrm{K}^{+}$ ions (as compared to $\mathrm{Li}^{+}$ions, for example) is responsible for the stronger pinning of water molecules on the surface [20].

A substrate that solves this surface stability problem, the (111) diamond surface partially terminated by alkali ions, was proposed recently [21]. Diamond is an interesting candidate for achieving extreme hydrophilic behavior on a substrate suitable for biomedical applications. It can be grown over large areas in polycrystalline form [22] and exhibits high corrosion resistance, making it attractive as a surface finish for biomedical devices and implants [23]. Moreover, its submonolayer surface structure has the added advantage of minimal lattice mismatch with ice Ih (less than $2 \%$ ), reducing any strain effects that could be detrimental to the stability of ice layers. Motivated by these considerations, we study here the stabilizing effect of the chemically modified diamond (111) surface on the melting transition of interfacial ice. We specifically consider the synthetically accessible [21] diamond surface in which $1 / 3$ of the $\mathrm{H}$ atoms in the original termination [denoted by $\mathrm{C}(111)-\mathrm{H}$ in the following] are replaced by $\mathrm{Na}$ atoms [denoted by $\mathrm{C}(111)-\mathrm{Na}$ ].

Direct simulations of the freezing phase transition are challenging [24], so we focus instead on understanding the stability of initially crystalline ice layers. We chose to use the TIP4P/Ice water model [25], in order to reproduce the melting temperature of ice at $1 \mathrm{~atm}$ and the stability of the Ih phase. We begin with a configuration in which eight bilayers [26] of ice Ih are aligned epitaxially with the $\mathrm{Na}$ atoms, which are covalently bonded to the $\mathrm{C}(111)$ surface, as shown in Fig. 1(a). The ice is initially completely polarized parallel to the $\mathrm{C}-\mathrm{Na}$ dipoles. The initial Na-O distance is $2.38 \AA$, as calculated previously [21]. An Anderson barostat [27,28] with a 100-fs relaxation time was used and two independent thermostats were maintained for the diamond and the ice multilayer to prevent unphysical heat transfer.

The interaction between atoms is represented by a Lennard-Jones (LJ) potential and a Coulomb potential with a 14.69- $\AA$ cutoff, using LJ parameters [29] for surface $H$ adapted from Ref. [30], surface Na from Ref. [31], and diamond $\mathrm{C}$ from Ref. [32]. The bond lengths [33] for C-Na are 
(a)

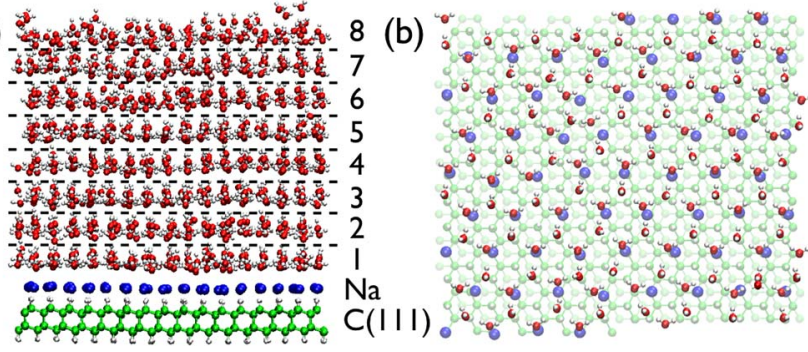

FIG. 1. (Color online) Visualization of epitaxial ice bilayers on the modified diamond surface. (a) Side view, showing the slab representing the $\mathrm{C}(111)$-Na substrate at the bottom and the eight initially distinct bilayers of ice separated by dashed lines in an instantaneous configuration after $5 \mathrm{ps}$ at $310 \mathrm{~K}$. The distance between dashed lines is $3.7 \AA$. (b) Top view of bilayer 1 with $\mathrm{C}(111)-\mathrm{Na}$ surface in background.

adapted from Ref. [21], and for C-C and C-H from Ref. [34]. The C(111) surface is represented by a $33.94 \AA \times 29.39 \AA$ periodic supercell in the lateral dimensions and two bilayers in the (111) direction, capped by $\mathrm{H}$ atoms on the far side from the ice. The $\mathrm{H}$ and $\mathrm{Na}$ atoms have partial charges of $+0.1 e$ and $+0.4 e$, respectively, as determined from $a b$ initio quantum mechanical calculations [21], which are neutralized by the directly bonded $\mathrm{C}$ atoms.

We turn next to the method of determining melting of the ice layers. In two-dimensional melting, mean square displacements diverge [35] and are not a good criterion to distinguish between liquid and solid states [36]. Instead, we adapted a shell-relative-displacement criterion [37] to intrabilayer neighbor shells: we define the local displacement

$$
\mathbf{u}_{i}(t)=\frac{1}{a}\left(\mathbf{r}_{i}(t)-\frac{1}{3} \sum_{j \in \mathrm{NN}} \mathbf{r}_{j}(t)\right)
$$

where $\mathbf{r}_{i}$ is the position of a water $\mathrm{O}$ atom $i$, the sum is taken over the positions of the three initial nearest-neighbor $\mathrm{O}$ atoms within the bilayer of that atom, and $a=2.75 \AA$ is the measured O-O separation in ice Ih [26]. The atomic Lindemann parameter is defined as the deviation

$$
s_{i}(t)=\left[\frac{1}{t+1} \sum_{l=0}^{t} \mathbf{u}_{i}^{2}(l)-\left(\frac{1}{t+1} \sum_{l=0}^{t} \mathbf{u}_{i}(l)\right)^{2}\right]^{1 / 2},
$$

where the sums are taken over all previous time steps. The Lindemann parameter for bilayer $n$ is then naturally defined as $\delta_{n}(t)=\left\langle s_{i}(t)\right\rangle$, where the average is over all $\mathrm{O}$ atoms in the bilayer. The displacements and Lindemann parameters defined above are independent of bilayer shear. This feature proved essential since, at $240 \mathrm{~K}$, bilayer 1 was observed to relax laterally from its initial epitaxial alignment such that, after $1 \mathrm{ps}$, the bottom-most $\mathrm{O}$ atoms close-packed between $\mathrm{Na}$ atoms. However, at $310 \mathrm{~K}$, bilayer 1 remained approximately epitaxial to the Na submonolayer, as shown in Fig. 1(b).

A dynamic criterion for determining if a bilayer has melted at a given temperature can be defined in terms of the dynamic Lindemann parameter $\delta_{n}(t)$. When bilayers melt, the motion of each water molecule becomes uncorrelated

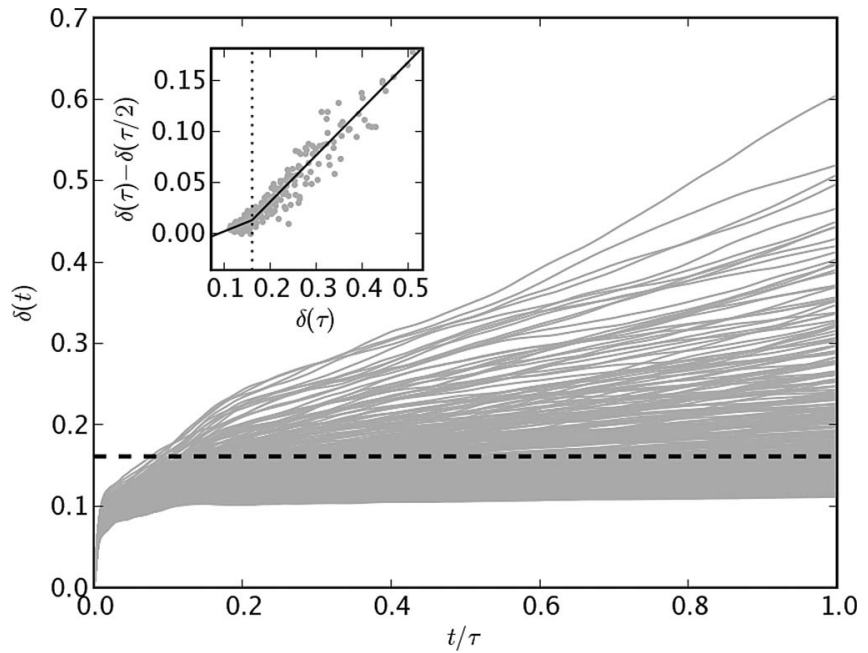

FIG. 2. Calculation of the bilayer melting threshold. Main figure: Time evolution of Lindemann parameters $\delta_{n}(t)$ for all ice bilayers over a range of temperatures. Inset: Extraction of $\delta_{L}$ from a piecewise linear fit of $\delta_{n}(\tau)$ versus $\delta_{n}(\tau)-\delta_{n}(\tau / 2)$. The fitted value of $\delta_{L}$ is indicated with dashed lines.

with the motion of its initial nearest neighbors, allowing the displacement $\mathbf{u}_{i}$ to diffuse freely, and causing $\delta_{n}(t)$ to grow linearly with time [36]. In contrast, for layers that have not yet melted, $\delta_{n}(t)$ saturates asymptotically to a constant value [36]. For sufficiently long simulation times $\tau$, this melting transition manifests as a first-order discontinuity in $\delta_{n}(\tau)$ versus $\delta_{n}(\tau)-\delta_{n}(\tau / 2)$, since $\delta_{n}(\tau)-\delta_{n}(\tau / 2)$ approaches zero asymptotically as a function of $\tau$ below the melting point. The critical $\delta_{n}(\tau)$ value separating the linearly growing versus constant values is then used as the effective Lindemann criterion $\delta_{L}[36]$.

With these definitions, we can extract a value for $\delta_{L}$ from the molecular dynamics simulations. By examining collectively the evolutions over time $\tau=5 \mathrm{ps}$ of all ice bilayers under a range $(240-340 \mathrm{~K})$ of ice thermostat values, we find $\delta_{L}=0.161 \pm 0.009$ (corresponding to a $0.44 \AA$ r.m.s. shell displacement), as shown in Fig. 2. This value agrees well with the threshold of 0.167 predicted by the cooperative motion model of 2D melting, in which layers slide past each other $[37,38]$. As additional confirmation, Lindemann parameters that rose above $\delta_{L}$ tended to continue to increase monotonically, while those below saturated. Finally, the ice vapor pressure was found to be nonzero when the Lindemann parameters of the top-most layers rose above $\delta_{L}$, as seen by the molecules about to be released from layer 8 in Fig. 1(a).

To solve for the melting point of each bilayer, $\delta_{L}$ must be equated to the Lindemann parameter, as a function of temperature, for that bilayer. Since $\delta_{n}(t)$ for a melted bilayer does not saturate with time, the Lindemann function was calculated after the finite time $\tau=5 \mathrm{ps}$. Cubic spline interpolation, with quadratic extrapolation for boundary temperatures, was then applied to each Lindemann function to solve for its unique melting point. The final parameters $\delta_{n}(\tau)$ are shown in Fig. 3 over the temperature range $210-350 \mathrm{~K}$, for all ice bilayers in three different environments: free ice, ice on $\mathrm{C}(111)-\mathrm{H}$, and ice on $\mathrm{C}(111)-\mathrm{Na}$. For $\delta(\tau)<\delta_{L}$, the func- 


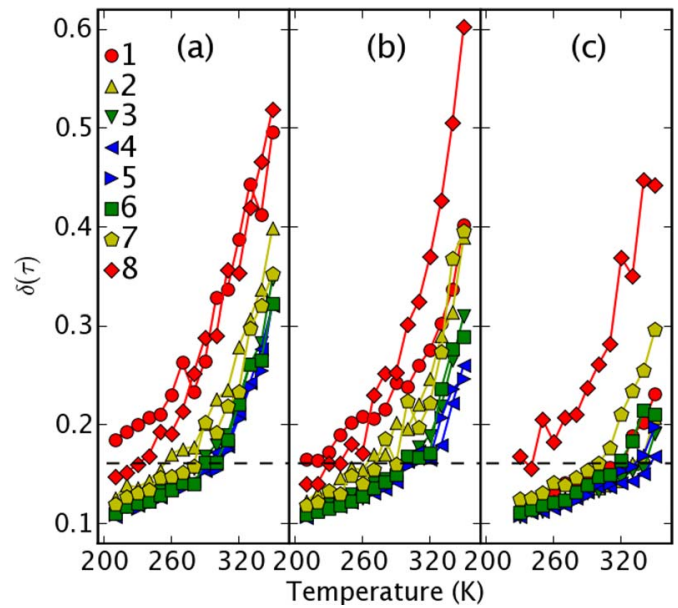

FIG. 3. (Color online) Temperature dependence of Lindemann parameters for ice bilayers in different environments. The environments considered are (a) free ice, (b) ice on C(111)-H, and (c) ice on $\mathrm{C}(111)-\mathrm{Na}$. The melting threshold $\delta_{L}$ is indicated with dashed lines.

tions $\delta(\tau)$ of temperature $T$ scale roughly with $T^{1 / 2}$, which is expected for diffusion in a harmonic potential. For $\delta(\tau)>\delta_{L}$, the functions scale linearly or superlinearly with temperature, which is suggestive of ballistic motion and energy transfer to melted molecules in those bilayers.

The calculated melting temperatures for bilayers in each environment are shown in Figs. 4(a)-4(c). The primary source of error in each melting curve is the uncertainty in $\delta_{L}$. We discuss first the melting curves in free ice and ice on $\mathrm{C}(111)-\mathrm{H}$, which are our reference systems, the first being devoid of any substrate effects, the second being a hydrophobic surface [39]. The melting curves for both environments show an approximate symmetry, with bilayers 1 and 8 melting at temperatures much lower (by $70-115 \mathrm{~K}$ ) than the

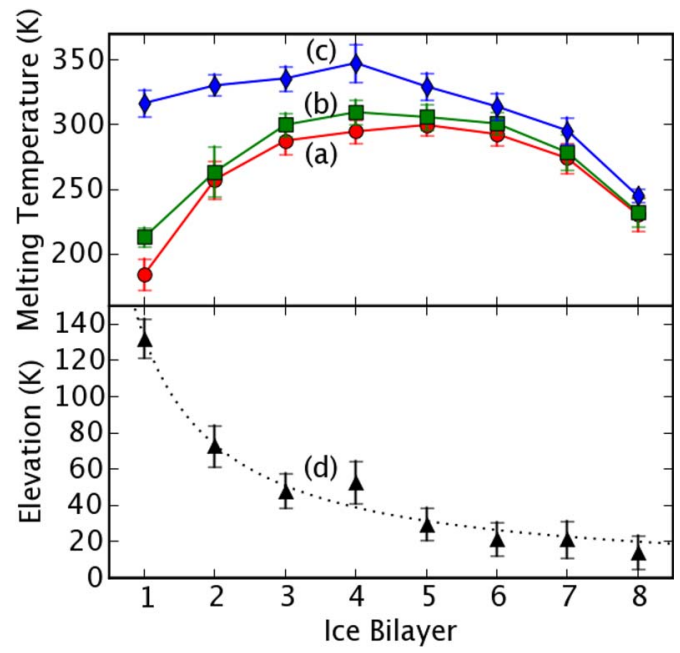

FIG. 4. (Color online) Calculated melting points for ice bilayers in different environments. Melting temperatures are shown for each bilayer in (a) free ice, (b) ice on $\mathrm{C}(111)-\mathrm{H}$, and (c) ice on $\mathrm{C}(111)-\mathrm{Na}$. (d) The melting point elevation of bilayers in ice on $\mathrm{C}(111)-\mathrm{Na}$ relative to free ice, with the phenomenological fit shown as a dotted line. melting temperature of the middle bilayers. The difference in melting temperature between layers 1 and 8 of $20-45 \mathrm{~K}$ is attributable to the polarization of the ice film, which exposes asymmetric ice surface structures. The close correspondence between the behavior of free ice and ice on $\mathrm{C}(111)-\mathrm{H}$ is consistent with the latter exhibiting hydrophobic behavior.

In sharp contrast to these two control environments, ice on $\mathrm{C}(111)-\mathrm{Na}$ shows an increase of the melting temperature by $130 \mathrm{~K}$ for bilayer 1 relative to free ice. Moreover, the increase in melting temperature persists through the entire ice film, as shown in Fig. 4(d), such that six bilayers $(2.2 \mathrm{~nm}$ thick) are predicted to remain frozen at $310 \mathrm{~K}$ (human body temperature) and seven bilayers ( $2.6 \mathrm{~nm}$ thick) remain frozen at $298 \mathrm{~K}$ (room temperature). The average melting temperature of free ice is $265 \pm 37 \mathrm{~K}$, in agreement with the established 1-atm value, and ice on $\mathrm{C}(111)-\mathrm{H}$ has only a moderately elevated average melting temperature of $275 \pm 34 \mathrm{~K}$. In contrast, ice on $\mathrm{C}(111)$ - Na has an average melting temperature of $314 \pm 30 \mathrm{~K}$.

We next develop a phenomenological model to explain the observed temperature elevation, by considering separately the electrostatic stabilization of bilayer 1 and the stabilization of each subsequent bilayer by the previous bilayer. Assume that each molecule in bilayer $n$ experiences a planar potential well $V_{n}(\mathbf{r}, z)=A_{n} \mathbf{r}^{2} \delta\left(z-z_{n}\right)$ centered at its equilibrium position in the ice phase. The Boltzmann position distribution is then given by

$$
P_{n}(\mathbf{r})=\frac{A_{n}}{\pi k_{B} T} \exp \left(-\frac{A_{n} \mathbf{r}^{2}}{k_{B} T}\right)
$$

Furthermore, if the planar potentials in bilayer $n>1$ are centered at the projected molecular positions in bilayer $n-1$, the individual thermal distributions are convolved, $P_{n}^{\prime}(\mathbf{r})=P_{1}(\mathbf{r}) * P_{2}(\mathbf{r}) * \ldots * P_{n}(\mathbf{r}) \propto \exp \left(-B_{n} \mathbf{r}^{2} / k_{B} T\right)$, with $B_{n}$ $\equiv\left(\sum_{i=1}^{n} A_{i}^{-1}\right)^{-1}$. Since the dipole-dipole interaction strength drops off as $z^{-3}$, we can neglect surface field effects for $n>1$ and assume that $A_{2}=A_{3}=\cdots=A_{n}$. Therefore, molecules in bilayer $n$ experience an additional effective potential from the diamond surface $V_{n}^{\prime}(\mathbf{r}, z)=\left[A_{1}^{-1}+(n-1) A_{2}^{-1}\right]^{-1} \mathbf{r}^{2} \delta\left(z-z_{n}\right)$. For a thermalized molecule in this effective potential $\left\langle\mathbf{r}_{n}^{2}\right\rangle$ $=\left[A_{1}^{-1}+(n-1) A_{2}^{-1}\right] k_{B} T$, and therefore the melting temperature at the Lindemann threshold $\left\langle\mathbf{r}_{n}^{2}\right\rangle \sim \delta_{L}^{2} a^{2}$ is given by

$$
T_{n}=\left[A_{1}^{-1}+(n-1) A_{2}^{-1}\right]^{-1} \delta_{L}^{2} a^{2} / k_{B} .
$$

The observed temperature elevation is reproduced well with $A_{1}=0.94 \mathrm{~J} / \mathrm{m}^{2}$ and $A_{2}=1.18 \mathrm{~J} / \mathrm{m}^{2}$, as shown in Fig. 4(d). To justify the value of $A_{1}$, we consider the strength of the potential in the equilibrium plane of a bilayer- 1 water molecule epitaxial to the diamond surface, due to near-field dipoledipole interaction with ions on the modified diamond surface. For surface charges $q=0.4 e$ with separation $d=2.5 \AA$, a nearly epitaxial water molecule with dipole moment $p$ parallel to the surface dipole experiences an in-plane quadratic potential $V_{1}(\mathbf{r}, z)=A_{1}^{\prime} \mathbf{r}^{2} \delta\left(z-z_{1}\right)$ with strength 


$$
A_{1}^{\prime}=\frac{3 p q}{8 \pi \epsilon_{0}}\left(\frac{1}{(z-d / 2)^{4}}-\frac{1}{(z+d / 2)^{4}}\right) .
$$

For submelting displacements at a distance $z$ away from the surface, with $z_{1} \leq z \leq z_{1}+\delta_{L}$, we obtain $A_{1}^{\prime}=1.04 \pm 0.26 \mathrm{~J} / \mathrm{m}^{2}$, in excellent agreement with the value obtained from the fit of Eq. (4). This suggests that the electrostatic interaction of molecules in bilayer 1 with the neighboring $\mathrm{Na}^{+}$sites dominates the observed melting temperature elevation for that bilayer.
In summary, we have shown through simulation that chemically modified diamond may stabilize, at elevated temperatures, ice multilayers substantially thicker than experimentally observed on other surfaces. This work opens up the possibility of protective ice finishes for in vivo diamond applications without cryogenics.

This work has been supported by the Fannie and John Hertz Foundation. We thank B. Halperin, M. Fyta, Sh. Meng, T. Sullivan, and J. Krich for helpful discussions.
[1] R. Lappalainen, A. Anttila, and H. Heinonen, Clin. Orthop. Relat. Res. 352, 118 (1998).

[2] S. Mitura, K. Zolynski, P. Witkowski, A. Kaluzny, Z. Has, and P. Niedzielski, J. Chem. Vap. Deposition 4, 232 (1996).

[3] D. P. Dowling, P. V. Kola, K. Donnelly, T. C. Kelly, K. Brumitt, L. Lloyd, R. Eloy, M. Therin, and N. Weill, Diamond Relat. Mater. 6, 390 (1997).

[4] J. Franks and D. Finch, Nanotechnology in Medicine and the Biosciences (Gordon and Breach Publishers, 1996).

[5] C. Du, X. W. Su, F. Z. Cui, and X. D. Zhu, Biomaterials 19, 651 (1998).

[6] G. Dearnaley, Clin. Mater. 12, 237 (1993).

[7] L. Tang, C. Tsai, W. W. Gerberich, L. Kruckeberg, and D. R. Kania, Biomaterials 16, 483 (1995).

[8] J. R. A. Freitas, Biocompatibility Vol. IIA of Nanomedicine, (Landes Bioscience, 2003).

[9] J. Hu, X.-D. Xiao, D. F. Ogletree, and M. Salmeron, Science 268, 267 (1995).

[10] P. B. Miranda, L. Xu, Y. R. Shen, and M. Salmeron, Phys. Rev. Lett. 81, 5876 (1998).

[11] M. Odelius, M. Bernasconi, and M. Parrinello, Phys. Rev. Lett. 78, 2855 (1997).

[12] X. Su, L. Lianos, Y. R. Shen, and G. A. Somorjai, Phys. Rev. Lett. 80, 1533 (1998).

[13] C.-Y. Ruan, V. A. Lobastov, F. Vigliotti, S. Chen, and A. H. Zewail, Science 304, 80 (2004).

[14] R. Zangi, J. Phys.: Condens. Matter 16, S5371 (2004).

[15] V. Ostroverkhov, G. A. Waychunas, and Y. R. Shen, Phys. Rev. Lett. 94, 046102 (2005).

[16] E.-M. Choi, Y.-H. Yoon, S. Lee, and H. Kang, Phys. Rev. Lett. 95, 085701 (2005).

[17] R. Zangi and A. E. Mark, J. Chem. Phys. 120, 7123 (2004).

[18] K. Koga, X. C. Zeng, and H. Tanaka, Phys. Rev. Lett. 79, 5262 (1997).

[19] L. Cheng, P. Fenter, K. L. Nagy, M. L. Schlegel, and N. C. Sturchio, Phys. Rev. Lett. 87, 156103 (2001).

[20] S.-H. Park and G. Sposito, Phys. Rev. Lett. 89, 085501 (2002).
[21] S. Meng, Z. Zhang, and E. Kaxiras, Phys. Rev. Lett. 97, 036107 (2006).

[22] H. Okushi, Diamond Relat. Mater. 10, 281 (2001).

[23] A. Dorner-Reisel, C. Schürer, G. Irmer, F. Simon, C. Nischan, and E. Müller, Anal. Bioanal. Chem. 374, 753 (2002).

[24] M. Matsumoto, S. Saito, and I. Ohmine, Nature (London) 416, 409 (2002).

[25] J. L. F. Abascal, E. Sanz, R. G. Fernndez, and C. Vega, J. Chem. Phys. 122, 234511 (2005).

[26] V. F. Petrenko and R. W. Whitworth, Physics of Ice (Oxford University Press, Oxford, 2002).

[27] H. C. Andersen, J. Chem. Phys. 72, 2384 (1980).

[28] K. Hinsen, J. Comput. Chem. 21, 79 (2000).

[29] The LJ potential for an atom pair $X-Y$ was approximated by averaging the diatomic potentials for $X-X$ and $Y-Y$. For the diatomic potential form, $U_{X-X}(r)=4 \epsilon_{X}\left[\left(\sigma_{X} / r\right)^{12}-\left(\sigma_{X} / r\right)^{6}\right]$ the parameters used to model the surface were $\epsilon_{\mathrm{H}}$ $=0.0157 \mathrm{kcal} / \mathrm{mol}, \sigma_{\mathrm{H}}=1.3248 \AA, \epsilon_{\mathrm{C}}=0.1094 \mathrm{kcal} / \mathrm{mol}, \sigma_{\mathrm{C}}$ $=1.6998 \AA, \epsilon_{\mathrm{Na}}=0.00277 \mathrm{kcal} / \mathrm{mol}, \sigma_{\mathrm{Na}}=1.6642 \AA$.

[30] W. L. Jorgensen and J. Tirado-Rives, J. Am. Chem. Soc. 110, 1657 (1988).

[31] J. Aqvist, J. Phys. Chem. 94, 8021 (1990).

[32] D. C. Spellmeyer and K. N. Houk, J. Org. Chem. 52, 959 (1987).

[33] The diamond surface bond lengths used were $1.5 \AA$ (C-C), $2.5 \AA(\mathrm{C}-\mathrm{Na})$, and $1.09 \AA(\mathrm{C}-\mathrm{H})$.

[34] S. J. Weiner, P. A. Kollman, D. T. Nguyen, and D. A. Case, J. Comput. Chem. 7, 230 (1986).

[35] J. Fröhlich and C. Pfister, Commun. Math. Phys. 81, 277 (1981).

[36] K. Zahn and G. Maret, Phys. Rev. Lett. 85, 3656 (2000).

[37] X. H. Zheng and J. C. Earnshaw, Europhys. Lett. 41, 635 (1998).

[38] B. J. Alder, W. G. Hoover, and T. E. Wainwright, Phys. Rev. Lett. 11, 241 (1963).

[39] Y. Kaibara, K. Sugata, M. Tachiki, H. Umezawa, and H. Kawarada, Diamond Relat. Mater. 12, 560 (2003). 